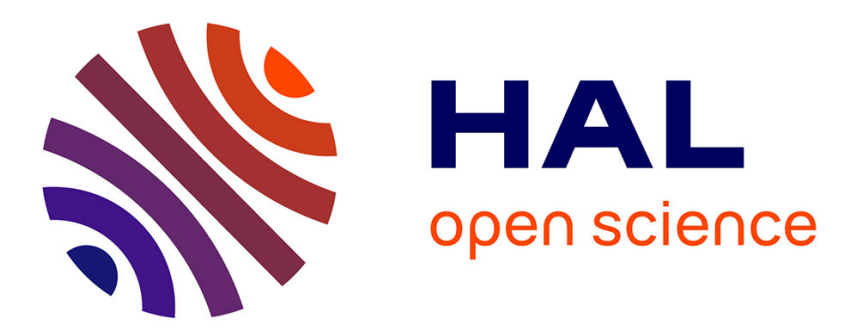

\title{
Control of soliton pattern through continuous external injection
}

\author{
Alioune Niang, Foued Amrani, Mohamed Salhi, Hervé Leblond, Andrey \\ Komarov, François Sanchez
}

\section{To cite this version:}

Alioune Niang, Foued Amrani, Mohamed Salhi, Hervé Leblond, Andrey Komarov, et al.. Control of soliton pattern through continuous external injection. SPIE Photonics Europe (Nonlinear Optics and Its Applications VIII; and Quantum Optics III), Apr 2014, Brussels, Belgique. pp.91361A, 10.1117/12.2050862 . hal-03204333

\section{HAL Id: hal-03204333 \\ https://univ-angers.hal.science/hal-03204333}

Submitted on 21 Apr 2021

HAL is a multi-disciplinary open access archive for the deposit and dissemination of scientific research documents, whether they are published or not. The documents may come from teaching and research institutions in France or abroad, or from public or private research centers.
L'archive ouverte pluridisciplinaire HAL, est destinée au dépôt et à la diffusion de documents scientifiques de niveau recherche, publiés ou non, émanant des établissements d'enseignement et de recherche français ou étrangers, des laboratoires publics ou privés. 
Return to the Manage Active Submissions page at http://spie.org/app/submissions/tasks.aspx and approve or disapprove this submission. Your manuscript will not be published without this approval. Please contact author_help@spie.org with any questions or concerns.

\title{
Control of soliton pattern through continuous external injection
}

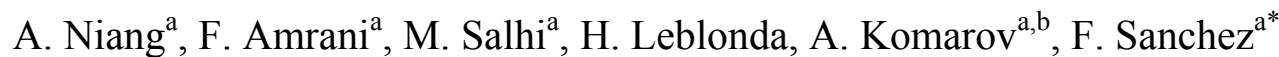 \\ ${ }^{a}$ Laboratoire de Photonique d'Angers E.A. 4464, Université d'Angers, 2 Bd Lavoisier, 49045 Angers \\ Cedex, France; ${ }^{b}$ Institute of Automation and Electrometry, Russian Academy of Sciences, Acad. \\ Koptyug Pr. 1, 630090 Novosibirsk, Russia
}

\begin{abstract}
The effect of an external continuous wave (cw) on the operating regime of a passively mode-locked double-clad fiber laser, operating in the anomalous dispersion regime, is experimentally investigated. Starting from different soliton distributions, we demonstrate that, under specific conditions, the $\mathrm{cw}$ signal forces the principal laser to operate in harmonic mode-locking regime.
\end{abstract}

Keywords: Fiber laser, mode-locking, optical injection

\section{INTRODUCTION}

Soliton interaction has now a long history since the initial papers in the middle 80's which concerned conservative solitons ${ }^{1,2}$. With the emergence of fiber lasers, and in particular double-clad fiber lasers, there was a revival on soliton interaction in the framework of dissipative soliton ${ }^{3-8}$. Indeed, in passively mode-locked high power fiber laser, a large number of solitons can coexist in the cavity when operating in the anomalous dispersion regime. As a general rule, the number of solitons in passively mode-locked fiber lasers increases when the pumping power grows ${ }^{9-11}$. Many different soliton patterns have been reported independently of the exact mode-locking mechanism revealing some universal properties $^{12-17}$. The resulting solitons distribution in fiber laser is a direct consequence of their interactions which can be repulsive or attractive or both at different scales. Attractive interaction is responsible of bound states ${ }^{5,18,19}$ or soliton crystals $^{20,21}$. Repulsive interaction is responsible of the well-known harmonic mode-locking ${ }^{22}$ (HML). In many HML fiber lasers, a continuous wave (cw) component is present in the optical spectrum suggesting that this component could play an important role in the HML mechanism ${ }^{23-26}$. Finally, one can note that although it is possible to easily generate different soliton patterns in a fiber laser by adjusting some external control parameter (in general it is a waveplate, a fibered polarization controller or merely the pumping power), a total control of the final distribution is not possible. Indeed the latter is reached after some evolution starting from noise, and generally depends on it, which is random. This differs from the evolution of pulses in an amplifier, and of several theoretical approaches in which suitable initial data are chosen to obtain a desired soliton distribution. It has been recently shown theoretically that a small cw component allows to control the nature and the strength of the soliton interaction ${ }^{27}$. Based on this prediction and on the role of the cw component in the HML, we have decided to conduct several series of experiments on a passively mode-locked fiber laser injected with an external cw component.

In this paper we demonstrate experimentally ${ }^{28}$ and theoretically ${ }^{29,30}$ for the first time that a passively mode-locked fiber laser can be forced to operate in HML regime by means of an external cw component. Starting from bound states, we show in section 2 that the external cw component can force the laser to operate in the harmonic mode-locking regime. Preliminary theoretical results are presented in section 3.

\section{EXPERIMENT}

\subsection{Experimental setup}

The experimental setup ${ }^{26}$ is schematically represented in Fig. 1. It is an all-fiber unidirectional ring cavity. Mode locking is achieved through nonlinear polarization evolution technique. We use a double-clad Er:Yb $10 \mathrm{~W}$ fiber amplifier operating at $\lambda=1.55 \mu \mathrm{m}$. It is pumped at $980 \mathrm{~nm}$ with several laser diodes injected with the v-groove technique. The

*francois.sanchez@univ-angers.fr; phone+33241735447 
Return to the Manage Active Submissions page at http://spie.org/app/submissions/tasks.aspx and approve or disapprove this submission. Your manuscript will not be published without this approval. Please contact author_help@spie.org with any questions or concerns.

maximum available pumping power is up to $40 \mathrm{~W}$ which ensures a total output power of amplified spontaneous emission up to $10 \mathrm{~W}$. The two fiber ends of the double-clad fiber are spliced to pieces of standard single-mode fibers (SMF28). The fibers DCF and SMF28 operate in the anomalous dispersion regime. A piece of dispersion-shifted fiber $\left(\beta_{2}^{D S F}=0.14 \mathrm{ps}^{2} / \mathrm{m}\right)$ is added to control the total cavity dispersion. To favor multiple-pulse mode locking, the total

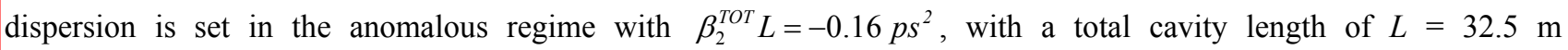
corresponding to a round trip time of $162.4 \mathrm{~ns}$ and to a free spectral range of $6.16 \mathrm{MHz}$. As usual, the adjustment of the polarization controllers allows obtaining a large variety of soliton distributions ${ }^{15}$. The external continuous wave is provided by a homemade cw tunable fiber laser and is launched with a 50/50 coupler to the principal cavity with a signal power that can be varied up to $800 \mathrm{~mW}$ (400 $\mathrm{mW}$ injected in the principal laser cavity). The external laser is tunable from $1530 \mathrm{~nm}$ to $1560 \mathrm{~nm}$ and its linewidth is about $1 \mathrm{~nm}$. For injection experiments, we processed as follows. First, the principal laser is switched on and the polarization controllers are adjusted to obtain some soliton pattern. While this pattern is stable, we switch on the external laser and then increase its wavelength starting from $1530 \mathrm{~nm}$. Its influence on the operating regime of the principal laser is studied. Reversibility of the phenomena is checked in different ways. Firstly, the wavelength of the external laser is decreased to retrieve its initial value and secondly, we reduce the injected power and switch off the injection laser.

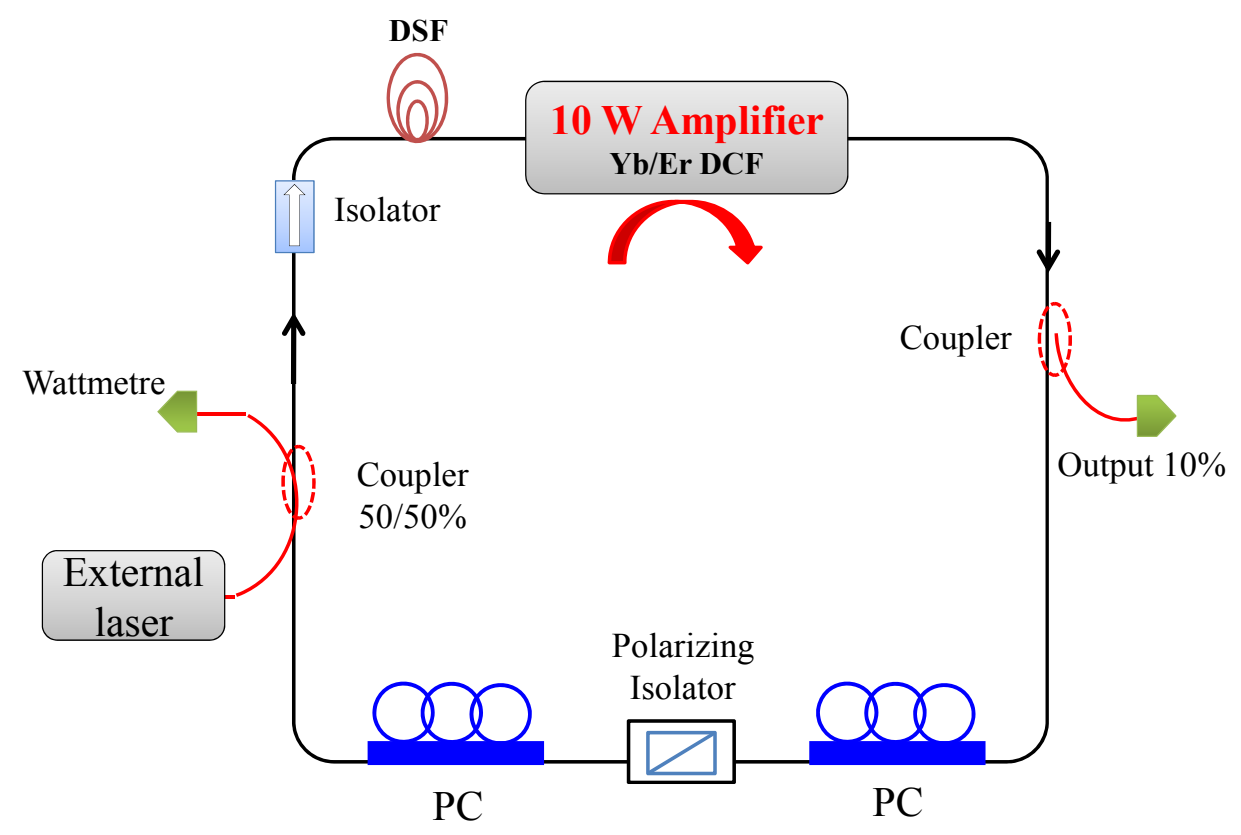

Figure 1. Experimental setup.

\subsection{Experimental results}

With a suitable adjustment of the polarization controllers we obtain the soliton pattern represented in Fig. 2(a). It consists in a set of well separated soliton packets which do not move from one round-trip to the other. Each packet contains a different number of solitons and repeat from round-trip to round-trip. The optical spectrum (not reported here) exhibits a modulation which is characteristic of a constant phase relation between the solitons, thus suggesting that the trains of solitons contain bound states ${ }^{19}$, or soliton crystals ${ }^{20}$ depending on the number of solitons involved in a given sequence. The spectral period is $0.2 \mathrm{~nm}$ which correspond to a temporal separation of 41 ps confirmed by autocorrelation measurements. The total number $N$ of solitons is estimated to be $N \cong 1500$. 
Return to the Manage Active Submissions page at http://spie.org/app/submissions/tasks.aspx and approve or disapprove this submission. Your manuscript will not be published without this approval. Please contact author_help@spie.org with any questions or concerns.
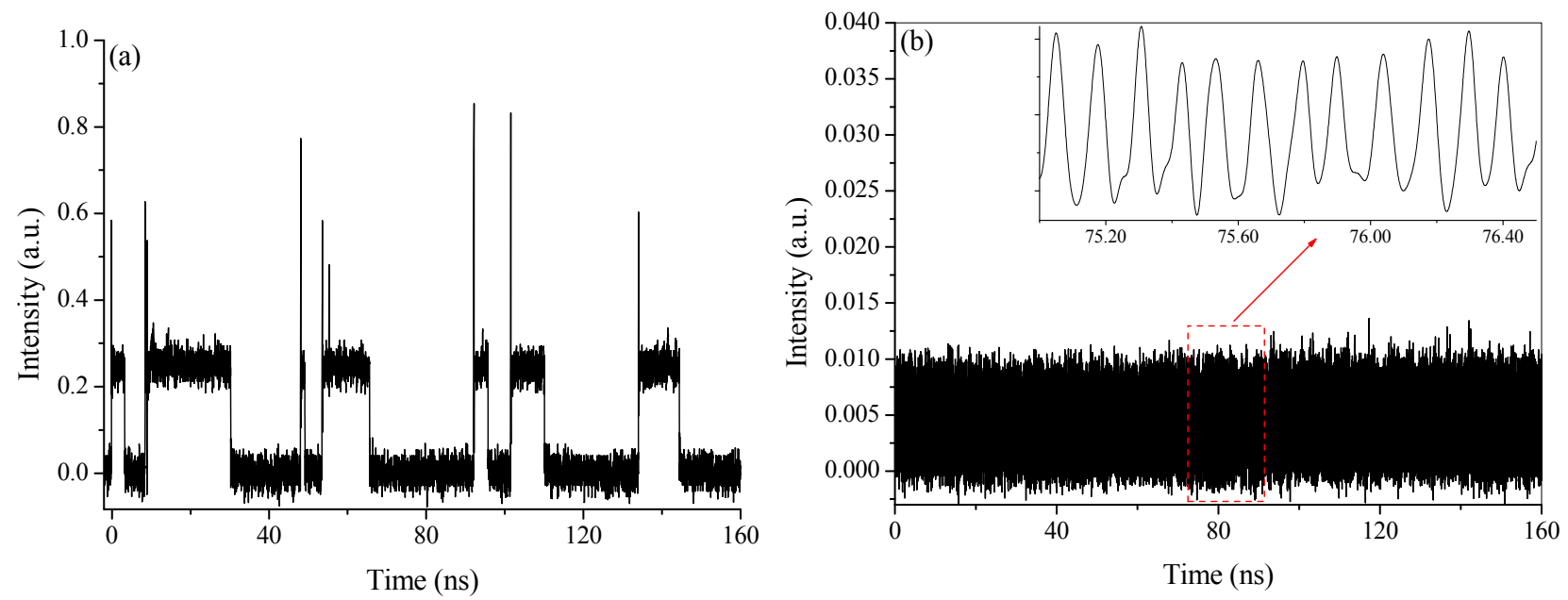

Figure 2. (a) Initial soliton distribution. (b) Final soliton distribution.

We then switched on the external laser with a start wavelength $\lambda_{\text {ext }}=1530 \mathrm{~nm}$ and with an injected power of $110 \mathrm{~mW}$ into the main laser cavity. The wavelength is then tuned towards longer values. While $\lambda_{e x t}$ enters the spectrum of the principal laser, the spectral modulation decreases, revealing that the $\mathrm{cw}$ component significantly modifies soliton interactions until it breaks the strong coupling of the solitons in a bound state ${ }^{31}$. In the temporal domain, the soliton packets become unstable: they move and collide to form larger condensed phases without evident internal order. While $\lambda_{\text {ext }}$ falls in the spectrum of the principal laser, pulses get loose from the condensed phases and span over the whole cavity. Solitons move like in a soliton gas and when $\lambda_{\text {ext }}$ moves toward the centre of the optical spectrum of the principal laser, the relative motion of the solitons decreases. When $\lambda_{\text {ext }}=1552 \mathrm{~nm}$, solitons stay at rest in the moving frame, nearly identical and equidistant as shown in Fig. 2(b). The optical spectrum given in Fig. 3(a) shows that there is no spectral modulation and, in addition to the cw component due to the injected laser $\left(\lambda_{\text {ext }}\right)$, there is an additional $\mathrm{cw}$ component generated by the principal laser $\left(\lambda_{\text {own }}\right)$ and characteristic of many passively HML fiber lasers ${ }^{24-26}$. The pulse duration, deduced from autocorrelation measurements, remains about 1 ps. HML is also pointed out through the radiofrequency spectrum of the recorded optical intensity, given in Fig. 3(b). The repetition rate of the laser is precisely 5.82 $\mathrm{GHz}$, which corresponds to the $945^{\text {th }}$ harmonics. The supermode suppression ratio is $12 \mathrm{~dB}$.
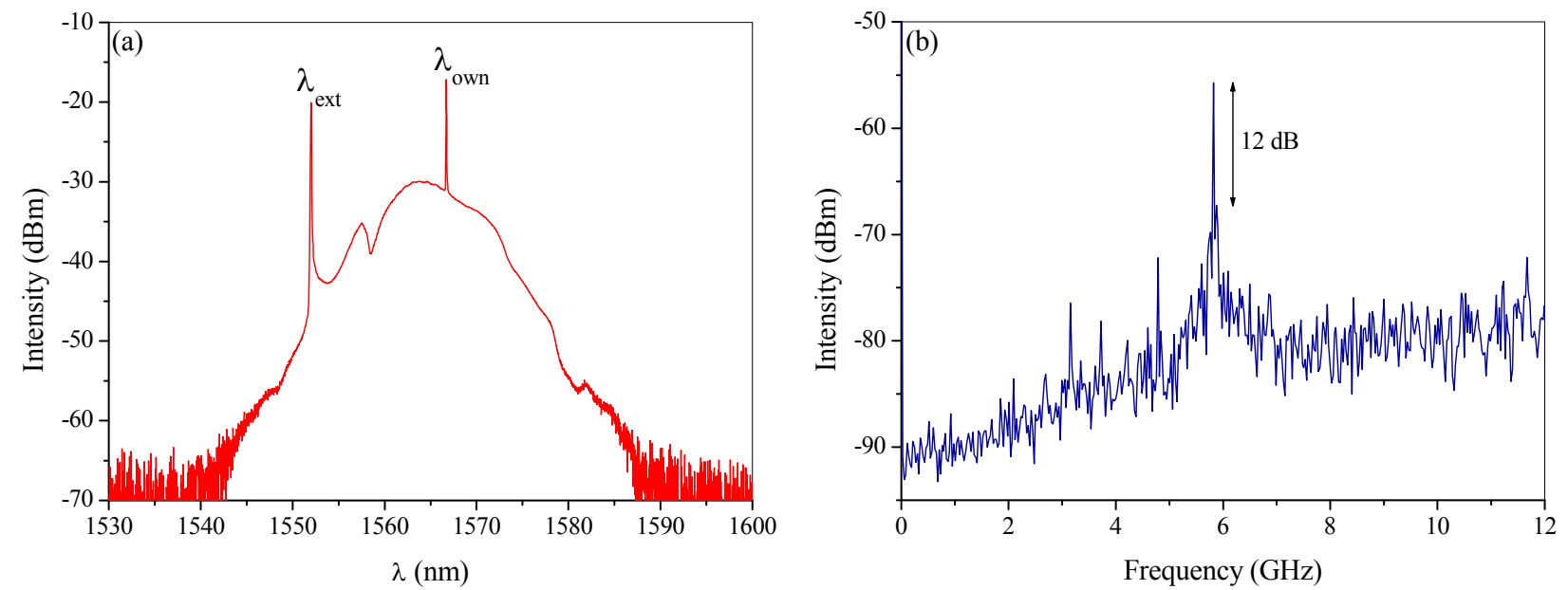

Figure 3. (a) Initial soliton distribution. (b) Final soliton distribution. 
Return to the Manage Active Submissions page at http://spie.org/app/submissions/tasks.aspx and approve or disapprove this submission. Your manuscript will not be published without this approval. Please contact author_help@spie.org with any questions or concerns.

If $\lambda_{\text {ext }}$ is further increased, the same HML regime persists until $\lambda_{\text {ext }}=1553 \mathrm{~nm}$. For longer values of $\lambda_{\text {ext }}$, the HML regime is lost and we obtain a regime where all solitons are in perpetual movement and fill the whole cavity: it is a soliton gas. If $\lambda_{\text {ext }}$ is now decreased, we observe the reverse scenario without any hysteresis phenomena: first the regime becomes HML and finally the initial soliton crystals and bound states are restored (same temporal trace, same optical spectrum and same autocorrelation trace). Hence, the effect of the external cw component is fully reversible. We have also verified that for $\lambda_{e x t}=1552 \mathrm{~nm}$, if the injected power is reduced, the HML regime persists in a wide range of injected powers. Indeed, HML occurs until the injected power reaches the lower value $50 \mathrm{~mW}$. Below this power, the initial soliton distribution is retrieved. If the injected power is increased again, the HML regime is restored. We are convinced that the $\mathrm{cw}$ component takes a key role in the self-organization of the pulses. Indeed, the initial bound state is formed thanks to the equilibrium between attraction and short distance repulsion. While the $\mathrm{cw}$ external field is tuned within the optical spectrum of the principal laser, this equilibrium is broken through the emergence of a repulsive interaction. When the condensed phases are destroyed, the pulses move under the effect of a long-range repulsion leading to the final soliton distribution (HML regime).

\section{THEORY}

Our theoretical approach considers the fiber laser represented in Fig. 4 in which the mode-locking is achieved through nonlinear polarization evolution. In addition to the Er-doped fiber amplifier, the laser resonator contains a polarization control system including the following sequentially arranged components: a half-wave phase plate with orientation angle $\alpha_{2}$ with respect to the $x$-axis, a quarter-wave plate (orientation angle $\alpha_{1}$, a polarizing isolator (the passing axis is parallel to the $x$-axis), and a second quarter-wave plate (orientation angle $\left.\alpha_{3}\right)^{10}$. The polarization control system produces nonlinear losses that form ultrashort pulses in the laser resonator.

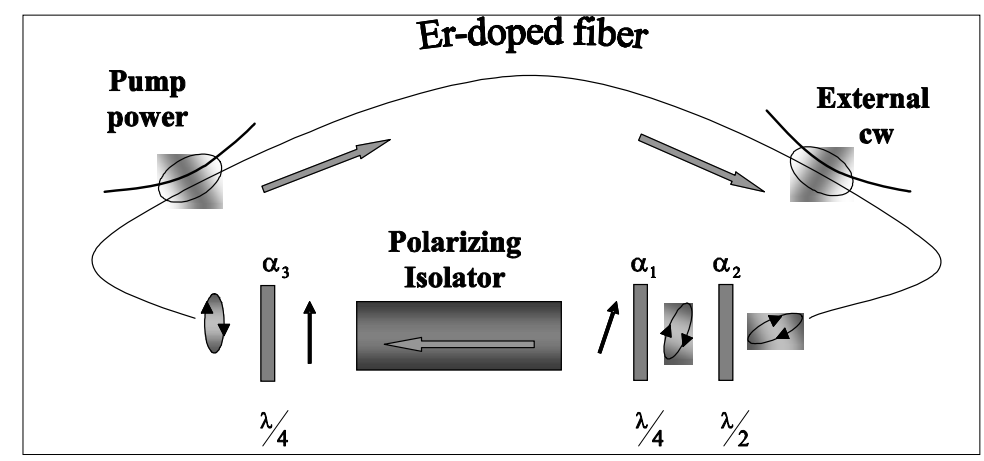

Figure 4. Schematic representation of a passive mode-locked erbium-doped fiber laser with a nonlinear polarization rotation technique and an external cw injection.

The evolution of the electric field components in the doped fiber is modeled with the vector model described by the following normalized equations ${ }^{10,29}$ :

$$
\begin{gathered}
\frac{\partial E_{x}}{\partial \varsigma}=\left(D_{r}+i D_{i}\right) \frac{\partial^{2} E_{x}}{\partial \tau^{2}}+g E_{x}+i q\left(\left|E_{x}\right|^{2} E_{x}+\frac{2}{3}\left|E_{y}\right|^{2} E_{x}+\frac{1}{3} E_{y}^{2} E_{x}^{*}\right)+P \exp (i \delta \omega \tau-i \delta \kappa \zeta), \\
\frac{\partial E_{y}}{\partial \varsigma}=\left(D_{r}+i D_{i}\right) \frac{\partial^{2} E_{y}}{\partial \tau^{2}}+g E_{y}+i q\left(\left|E_{y}\right|^{2} E_{y}+\frac{2}{3}\left|E_{x}\right|^{2} E_{y}+\frac{1}{3} E_{x}^{2} E_{y}^{*}\right),
\end{gathered}
$$

where $E_{x}$ and $E_{y}$ are two orthogonal components of the electric field expressed in unites $(\sqrt{\gamma L})^{-1}$ (here $L(m)$ is the dimensional cavity length and $\gamma\left(W^{-1} m^{-1}\right)$ is the dimensional nonlinear refractive coefficient related to the nonlinear refractive index coefficient ${ }^{10}$ ), $\zeta$ is the propagation distance (the number of passes of the radiation through the laser cavity), $\tau$ is the time coordinate in units $\delta t=\sqrt{\left|\beta_{2}\right| L / 2}$ (here $\beta_{2}$ is the second-order group-velocity dispersion for the 
Return to the Manage Active Submissions page at http://spie.org/app/submissions/tasks.aspx and approve or disapprove this submission. Your manuscript will not be published without this approval. Please contact author_help@spie.org with any questions or concerns.

intracavity medium), $D_{r}$ is the frequency dispersion of the gain and the linear losses, $D_{i}$ is the frequency dispersion of the refractive index, $q$ is the normalized nonlinearity of the refractive index, and $I=\left|E_{x}\right|^{2}+\left|E_{y}\right|^{2}$. The term $g$ describes the amplification $g=a /\left(1+b \int I d \tau\right)$, where the integration is carried out over the whole round-trip period, $a$ is the pumping parameter, and $b$ is the saturation parameter. The last term in Eq. (1) describes the electric-dipole polarization of the intracavity medium that produces the monochromatic radiation inside the laser cavity. By this means, we model the injected radiation inside the laser cavity ${ }^{29}$. The parameters $\delta \omega$ and $\delta \kappa$ are determined by the frequency and wave vector of the external injected monochromatic radiation. Here $\delta \omega$ is the detuning of the carrier frequency of the external radiation from the center of the spectral gain band. The parameters $\delta \omega$ and $\delta \kappa$ are chosen so that the injected radiation coincides with the radiation of one of the longitudinal modes of the laser resonator. This implies that $\delta \kappa$ and $\delta \omega$ satisfy the dispersion relation $\delta \kappa=D_{i}(\delta \omega)^{2}$, which is obtained from Eq. (1). In addition, for this purpose in our numerical simulation we choose the frequency detuning in the form $\delta \omega=2 \pi K / l$, where the frequency parameter $K$ is an integer and $l$ is the length of the temporal computation box. The direction of a polarization of the linearly polarized injected $\mathrm{cw}$ coincides with the direction of a $x$-axis. Varying the parameters $P$ and $K$, we can change the intensity $I_{c w}$ of the intracavity injected $\mathrm{cw}$ and its frequency detuning $\delta \omega$.

Let us first consider the movement of a soliton submitted to a cw external wave. Figure 5 shows the dependence of the soliton velocity $\delta v=d \tau / d \zeta$ on the detuning parameter $K$ of the injected cw. For the principle inverse peak in the vicinity $K=40$, the change of the phase difference of the soliton and the $\mathrm{cw}$ over one round-trip period is equal zero. Here the condition of the phase locking is fulfilled and the value $|\delta v|$ is large. Outside the phase-locking region, the phase difference for the soliton and the cw oscillates and the value $|\delta v|$ becomes small. For other peaks in Fig. 5 the change of the phase difference for the soliton and the cw over one round-trip period is a multiple of $2 \pi$. Figure 6 shows the dependence of the soliton velocity on the intensity $I_{c w}$ of the injected cw. Phase locking of the soliton and the injected cw occurs in the intensity interval between points 1 and 3. To the left of the point 1, phase locking is absent. To the right of the point 3 , the injected cw destroys the single soliton operation. Between points 1 and 2 as well as between points 2 and 3 the dependence of $\delta v$ on $I_{c w}$ is monotonic.

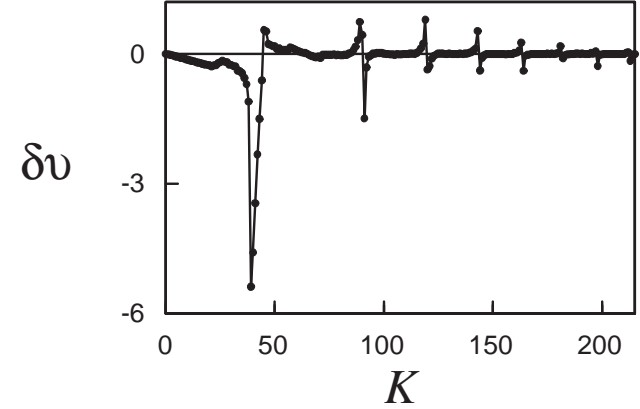

Figure 5. Soliton velocity $\delta v$ vs the frequency parameter $K$ of the injected $\mathrm{cw}$.

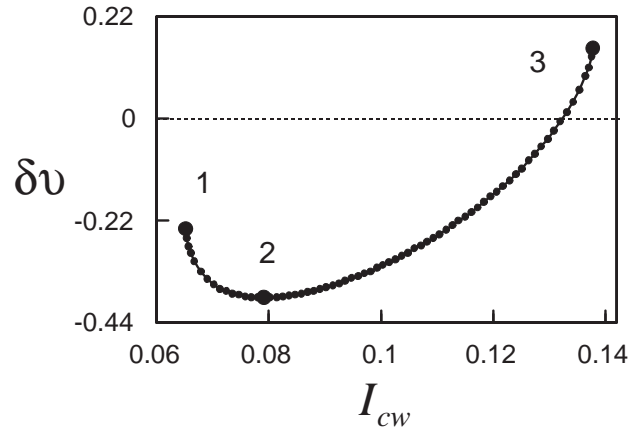

Figure 6. Soliton velocity $\delta v$ vs the intensity $I_{c w}$ of the injected cw. $K=40$.

Figure 7(a) shows a repulsion of laser solitons. After each pulse, the injected cw is attenuated because of its destructive interference with the right-wing dispersive waves. As a consequence, the resultant $\mathrm{cw}$ increases monotonically from left to right along the pulse train. The changing intensity of the resultant $\mathrm{cw}$ is between points 1 and 2 in Fig. 20, where the velocity of motion of pulses in the right direction increases monotonically with increasing intensity $I_{c w}$. As a result, the velocity of the solitons motion in the right direction increases monotonically along the soliton train from left to right. This distribution of soliton velocities in the train results in an increase of inter-soliton spacing, that is, to repulsion of the solitons. In this case, after a transient process the harmonic passive mode locking (multi-pulse operation with equidistant pulses) is realized (see Fig. 7(b)). Figure 8 demonstrates a formation of a soliton crystal through the light-induced drift of 
Return to the Manage Active Submissions page at http://spie.org/app/submissions/tasks.aspx and approve or disapprove this submission. Your manuscript will not be published without this approval. Please contact author_help@spie.org with any questions or concerns.

solitons in a laser cavity. The initial field is chosen in the form of a pair of strongly bound solitons and several individual solitons. The light-induced velocities of the soliton pair and an individual soliton are different. As a result, individual solitons drift toward the soliton pair, collide with it, stop and create a condensed soliton phase in the form of a soliton crystal.

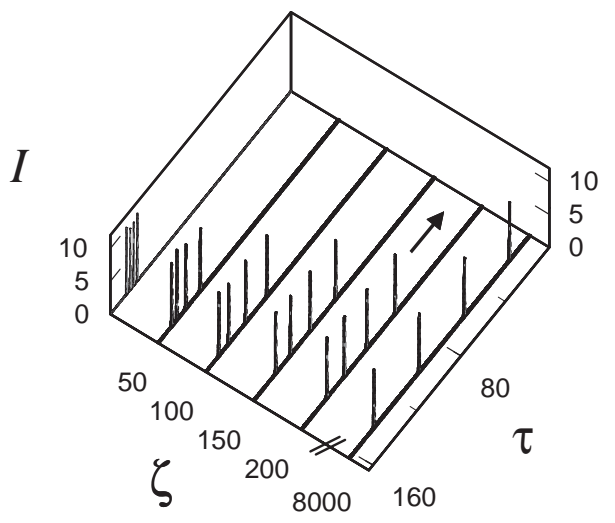

(a)

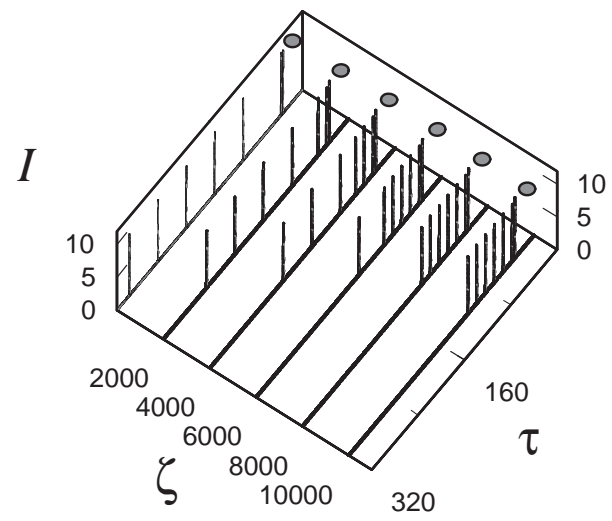

(a)

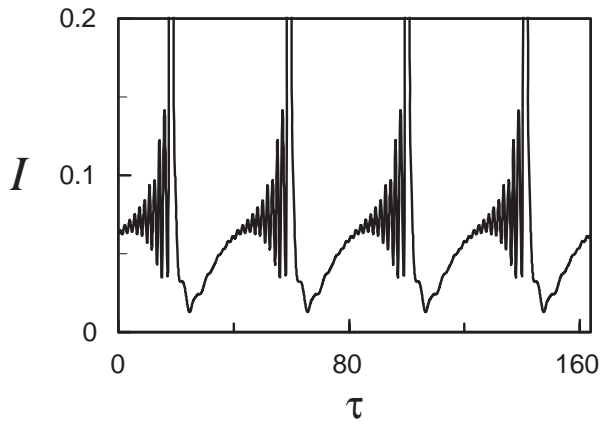

(b)

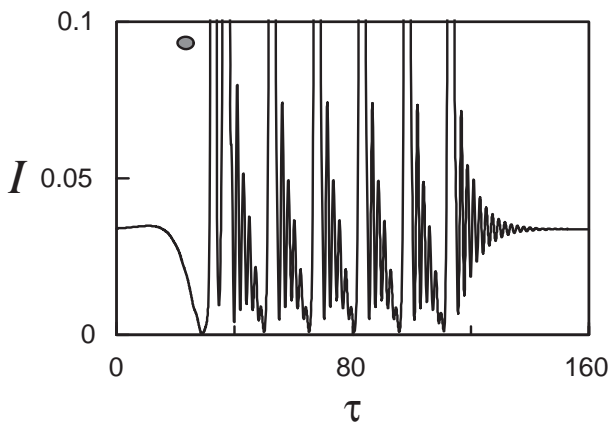

(b)

Figure 7. Dependence of the intensity distribution $I(\tau)$ on the number of round trips $\zeta$ in a passive mode-locked laser with an injected cw. Regime of a soliton repulsion. (a) Transient process. (b) Steady state (the scale is enlarged). $K=40$, $P=0.1, l \approx 164$.

Figure 8. Dependence of the intensity distribution $I(\tau)$ on the number of round trips $\zeta$ in a passive mode-locked laser with an injected cw. Regime of a soliton crystal. (a) Transient process. (b) Steady state (the scale is enlarged). The twosoliton molecule is marked by the grey circle. $K=76$, $P=0.09, l \approx 328$.

\section{CONCLUSION}

In this paper, we have investigated both experimentally and theoretically the effect of an external cw signal on the soliton distribution in a passively mode-locked fiber laser operating in the soliton regime. The main result is that harmonic mode-locking operation can be forced by the external injected wave.

\section{Acknowledgements}

This work was supported by the Agence Nationale de la Recherche (Contract No. ANR-2010-BLANC-0417-01SOLICRISTAL) and the Grant of Leading Scientific Schools of Russia No. 3753.2014.2. 
Return to the Manage Active Submissions page at http://spie.org/app/submissions/tasks.aspx and approve or disapprove this submission. Your manuscript will not be published without this approval. Please contact author_help@spie.org with any questions or concerns.

\section{REFERENCES}

[1] J. P. Gordon, "Interaction forces among solitons in optical fibers" Opt. Lett. 8, 596-598 (1983).

[2] F. M. Mitschke and L. F. Mollenauer, "Experimental observation of forces between solitons in optical fibers" Opt. Lett. 12, 355-357 (1987).

[3] B. A. Malomed, "Bound solitons in the nonlinear Schrödinger-Ginzburg-Landau equation" Phys. Rev. A 44, 6954-6957 (1991).

[4] V. V. Afanasjev, B. A. Malomed and P. L. Chu, "Stability of bound states of pulses in the Ginzburg-Landau equations" Phys. Rev. E 56, 6020-6025 (1997).

[5] N. N. Akhmediev, A. Ankiewicz and J. M. Soto-Crespo, "Multisoliton solutions of the complex GinzburgLandau equation" Phys. Rev. Lett. 79, 4047-4051 (1997).

[6] N. N. Akhmediev, A. Ankiewicz and J. M. Soto-Crespo, "Stable soliton pairs in optical transmission lines and fibre lasers" J. Opt. Soc. Am. B 15, 515-523 (1998).

[7] H. Leblond, A. Komarov, M. Salhi, A. Haboucha and F. Sanchez, "Cis bound states of three localized pulses of the cubic-quintic CGL equation" J. Opt. A: Pure Appl. Opt. 8, 319-326 (2006).

[8] A. Komarov, A. Haboucha, K. Komarov, H. Leblond, M. Salhi and F. Sanchez, "Soliton interaction in fiber lasers" Recent Research Developments in Optics, Ed. Dr. S.G. Pandalai 7, 63-112 (2009).

[9] D. Y. Tang, L. M. Zhao, B. Zhao and A. Q. Liu, "Mechanism of multisoliton formation and soliton energy quantization in passively mode-locked fiber laser" Phys. Rev. A 72, 043816 (2005).

[10] A. Komarov, H. Leblond and F. Sanchez, "Multistability and hysteresis phenomena in passively mode-locked fibre lasers" Phys. Rev. A 71, 053809 (2005).

[11] X. Liu, "Hysteresis phenomena and multipulse formation of a dissipative system in a passively mode-locked fiber laser" Phys. Rev. A 81, 023811 (2010).

[12] A. Haboucha, H. Leblond, M. Salhi, A. Komarov and F. Sanchez, "Coherent pattern soliton formation in a fiber laser" Opt. Lett._33, 524-526 (2008).

[13] D. Y. Tang, B. Zhao, L. M. Zhao and H. Y. Tam, "Soliton interaction in a fiber ring laser" Phys. Rev. E 72, $016616(2005)$.

[14] F. Amrani, A. Haboucha, M. Salhi, H. Leblond, A. Komarov and F. Sanchez, "Dissipative solitons compounds in a fiber laser: analogy with the states of the matter" Appl. Phys. B 99, 107-114 (2010).

[15] F. Amrani, M. Salhi, H. Leblond, Ph. Grelu and F. Sanchez, "Universal soliton pattern formation in passively mode-locked fiber lasers" Opt. Lett. 36, 1545-1547 (2011).

[16] A. Zaviyalov, R. Iliew, O. Egorov and F. Lederer, "Multi-soliton complexes in mode-locked fiber lasers" Appl. Phys. B 104, 513-521 (2011).

[17] Ph. Grelu and J. M. Soto-Crespo, "Multisoliton states and pulse fragmentation in a passively mode-locked fibre laser” J. Opt. B: Quantum Semiclass. Opt. 6, S271-S278 (2004).

[18] Y. D. Gong, P. Shum, T. Hiang, Cheng, Q. Wen and D. Y. Tang, "Bound soliton pulses in passively modelocked fiber laser" Opt. Com. 200, 389-399 (2001).

[19] Ph. Grelu, F. Belhache, F. Gutty and J. M. Soto-Crespo, "Phase-locked soliton pairs in a stretched-pulsed fiber laser" Opt. Lett. 27, 966-968 (2002).

[20] A. Haboucha, H. Leblond, M. Salhi, A. Komarov and F. Sanchez, "Analysis of soliton pattern formation in passively mode-locked fiber laser" Phys. Rev. A 78, 043806 (2008).

[21] A. Komarov, A. Haboucha and F. Sanchez, "Ultrahigh repetition rate bound-soliton harmonic passive modelocked laser" Opt. Lett. 33, 2254-2256 (2008).

[22] A. B. Grudinin and S. Gray, "Passive harmonic mode locking in soliton fiber lasers" J. Opt. Soc. Am. B. 14, 144-154 (1997).

[23] F. Amrani, A. Haboucha, M. Salhi, H. Leblond, A. Komarov, Ph. Grelu and F. Sanchez, "Passively modelocked erbium-doped double-clad fiber laser operating in the 322th harmonic" Opt. Lett. 34, 2120-2122 (2009).

[24] G. Sobon, K. Krzempek, P. Kaczmarek, K. M. Abramski and M. Nikodem, "10 GHz passive harmonic modelocking in Er-Yb double-clad fiber laser" Opt. Com. 284, 4203-4206 (2011).

[25] Z. X. Zhang, L. Zhan, X. X. Yang, S. Y. Luo and Y. X. Xia, "Passive harmonically mode-locked erbiumdoped fiber laser with scalable repetition rate up to 1.2 GHz" Laser Phys. Lett. 4, 592-596 (2007).

[26] F. Amrani, A. Niang, M. Salhi, H. Leblond and F. Sanchez, "Passive harmonic mode locking of soliton crystals" Opt. Lett. 36, 4239-4241 (2011). 
Return to the Manage Active Submissions page at http://spie.org/app/submissions/tasks.aspx and approve or disapprove this submission. Your manuscript will not be published without this approval. Please contact author_help@spie.org with any questions or concerns.

[27] A. Komarov, K. Komarov, H. Leblond and F. Sanchez, "Spectral-selective management of dissipative solitons in passive mode-locked fibre lasers" J. Opt. A: Pure Appl. Opt. 9, 1149-1156 (2007).

[28] A. Niang, F. Amrani, M. Salhi, H. Leblond, A. Komarov and F. Sanchez, "Harmonic mode-locking in a fiber laser through continuous external optical injection" Opt. Com. 312, 1-6 (2014).

[29] A. Komarov, K. Komarov, A. Niang and F. Sanchez, "Nature of soliton interaction in fiber lasers with continuous external injection" Phys. Rev. A 89, 013833 (2014).

[30] H. Leblond, A. Niang, F. Amrani, M. Salhi and F. Sanchez, "Motion of solitons of the complex GinzburgLandau equation: the effect of an external oscillating source" Phys. Rev. A 88, 033809 (2013).

[31] A. Komarov, K. Komarov and F. Sanchez, "Quantization of binding energy of structural solitons in passive mode-locked fiber lasers" Phys. Rev. A 79, 033807 (2009). 